\title{
BMJ Open Kidney disease and risk of dementia: a Danish nationwide cohort study
}

\author{
Alisa D Kjaergaard (D) , ${ }^{1,2}$ Benjamin R Johannesen, ${ }^{2}$ Henrik T Sørensen, ${ }^{2,3}$ \\ Victor W Henderson (iD) , ${ }^{2,4}$ Christian F Christiansen (iD ${ }^{2}$
}

To cite: Kjaergaard $A D$, Johannesen BR, Sørensen HT, et al. Kidney disease and risk of dementia: a Danish nationwide cohort study. BMJ Open 2021;11:e052652. doi:10.1136/ bmjopen-2021-052652

- Prepublication history and additional supplemental material for this paper are available online. To view these files, please visit the journal online (http://dx.doi.org/10.1136/ bmjopen-2021-052652).

Received 21 April 2021 Accepted 06 October 2021

Check for updates

(c) Author(s) (or their employer(s)) 2021. Re-use permitted under CC BY-NC. No commercial re-use. See rights and permissions. Published by BMJ.

${ }^{1}$ Steno Diabetes Center Aarhus, Aarhus University Hospital,

Aarhus, Denmark

${ }^{2}$ Department of Clinical

Epidemiology, Aarhus University Hospital, Aarhus, Denmark

${ }^{3}$ Excellence Research Center, Stanford University, Stanford, California, USA

${ }^{4}$ Departments of Epidemiology and Population Health and of Neurology and Neurological Sciences, Stanford University, Stanford, California, USA

Correspondence to

Dr Alisa D Kjaergaard;

alisa.kjaergaard@auh.rm.dk

\begin{abstract}
Objectives It is unclear whether kidney disease is a risk factor for developing dementia. We examined the association between kidney disease and risk of future dementia.
\end{abstract}

Design and setting Nationwide historical registry-based cohort study in Denmark based on data from 1 January 1995 until 31 December 2016.

Participants All patients diagnosed with kidney disease and matched general population cohort without kidney disease (matched 1:5 on age, sex and year of kidney disease diagnosis).

Primary and secondary outcome measures All-cause dementia and its subtypes: Alzheimer's disease, vascular dementia and other specified or unspecified dementia. We computed 5-year cumulative incidences (risk) and hazard ratios (HRs) for outcomes using Cox regression analyses. Results The study cohort comprised 82690 patients with kidney disease and 413405 individuals from the general population. Five-year and ten-year mortality rates were twice as high in patients with kidney disease compared with the general population. The 5-year risk for all-cause dementia was $2.90 \%$ (95\% confidence interval: $2.78 \%$ to $3.08 \%$ ) in patients with kidney disease and $2.98 \%$ (2.92\% to $3.04 \%)$ in the general population. Compared with the general population, the adjusted HRs for all-cause dementia in patients with kidney disease were 1.06 (1.00 to 1.12) for the 5-year follow-up and 1.08 (1.03 to 1.12) for the entire study period. Risk estimates for dementia subtypes differed substantially and were lower for Alzheimer's disease and higher for vascular dementia. Conclusions Patients diagnosed with kidney disease have a modestly increased rate of dementia, mainly driven by vascular dementia. Moreover, patients with kidney disease may be underdiagnosed with dementia due to high mortality and other comorbidities of higher priority.

\section{INTRODUCTION}

Dementia is a common, progressive agerelated neurological disorder diagnosed when acquired cognitive impairment has become severe enough to compromise social and/or occupational functioning. ${ }^{1}$ Although the incidence rates of dementia have decreased modestly over the last 30 years, the prevalence of dementia is increasing worldwide, likely due to increased life expectancy. ${ }^{2}$ This has enormous costs for the individuals

\section{Strengths and limitations of this study}

This is the first European population-based study examining the association between hospital-diagnosed kidney disease and risk of future dementia.

- We conducted a nationwide registry-based cohort study of all Danish residents with kidney disease and a 1:5 matched general population comparison cohort without kidney disease during a study period from 1995 to 2016.

- We did not have data on albuminuria or estimated glomerular filtration rate.

- Not all individuals with kidney disease or dementia are hospital diagnosed and thus captured in the Danish registries.

- Results pertaining to dementia subtypes should be interpreted cautiously due to potential differential misclassification of dementia subtypes, particularly among patients with kidney disease

and families affected, as well as the healthcare and society. ${ }^{3}$

Kidney disease is another disorder with a high (close to 10\%) and increasing prevalence, partly due to ageing population, and increased incidence rates of hypertension and diabetes mellitus. ${ }^{4}$

Kidney disease and dementia share risk factors such as increasing age, hypertension, diabetes mellitus, hyperlipidaemia and the pathophysiology of small vessel disease ${ }^{56}$ One potential link between kidney disease and dementia could be common susceptibility of kidney and brain tissue to vascular injury. ${ }^{7}$ Kidney disease is associated with oxidative stress, chronic inflammation and changes in coagulation, and it might also affect the brain or cerebral vasculature indirectly or directly through metabolic derangements and uremic toxins. 7

A previous population-based study in Taiwan found a hazard ratio (HR) of 1.41 for all-cause dementia in patients with a diagnosis of kidney disease ( $\mathrm{N}=37049)$ compared with the general population $(\mathrm{N}=74098){ }^{8}$ However, these findings may not be applicable to European populations, and the 


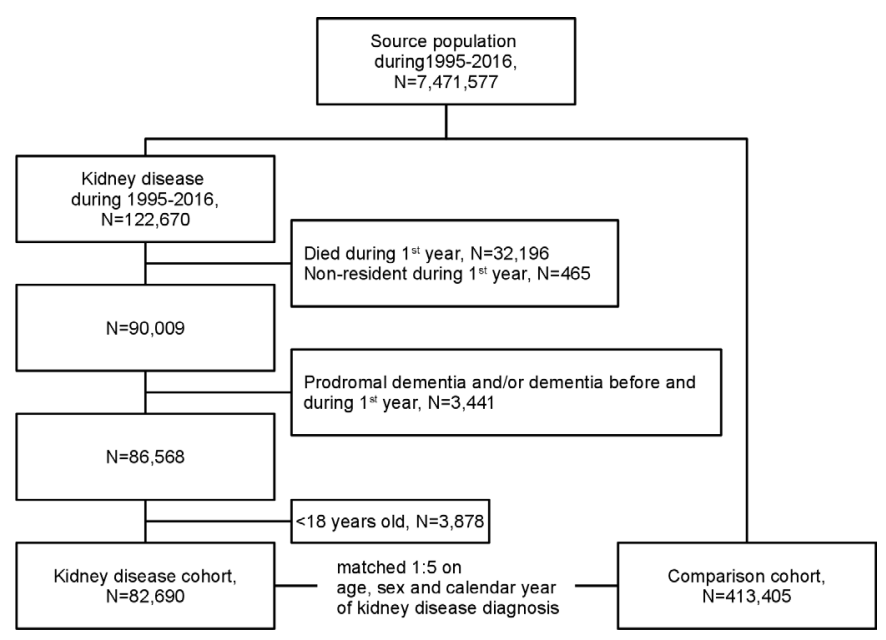

Figure 1 Study flow chart. Cohort of patients with incident kidney disease and individuals of the matched general population comparison cohort during 1995-2016.

Taiwanese study did not examine potential differences across dementia subtypes. Furthermore, previous studies, where kidney disease was defined as persistent albuminuria or estimated glomerular filtration rate (eGFR) below $60 \mathrm{~mL} / \mathrm{min} / 1.73 \mathrm{~m}^{2}$, reported mixed results. ${ }^{9-13}$ Thus, whether kidney disease is associated with risk of dementia is presently uncertain. We investigated this for all-cause dementia and dementia subtypes (Alzheimer's disease, vascular dementia and other dementia) in a nationwide cohort study.

\section{METHODS}

We followed the Strengthening the Reporting of Observational Studies in Epidemiology guidelines for reporting of cohort studies in epidemiology.

\section{Study cohort}

We conducted a nationwide cohort study of all Danish patients with hospital-diagnosed kidney disease and a matched general population comparison cohort without kidney disease during a study period from 1 January 1995 until 31 December 2016.

A flow chart of the study cohort is shown in figure 1. We identified 122670 patients with a first-time kidney disease diagnosis recorded during the study period. Next, we excluded patients who died $(\mathrm{N}=32196)$ or did not reside in Denmark $(\mathrm{N}=465)$ during the first year after kidney disease diagnosis. Further exclusion criteria were diagnosis of dementia $(\mathrm{N}=1909)$ and prodromal signs of dementia, that is, mild cognitive impairment and amnestic syndrome $(\mathrm{N}=303)$ before kidney disease diagnosis. Additionally, we excluded patients diagnosed with dementia $(\mathrm{N}=1300)$ and prodromal signs of dementia $(\mathrm{N}=156)$ during first year after a kidney disease diagnosis, because dementia diagnosed in this period is unlikely to be a consequence of kidney disease. Finally, we limited the cohort to adult patients aged 18 and above. The remaining 82690 patients comprised our kidney disease cohort. For each patient in the kidney disease cohort, up to five individuals from the general population without a kidney disease diagnosis prior to index date were randomly selected and matched on age (birth year), sex and calendar year of index date, that is, date of kidney disease diagnosis. Matching was performed as individual matching with replacement. ${ }^{14}$ The general population comparison cohort comprised 413405 individuals, who were alive and had no dementia, mild cognitive impairment, amnestic syndrome or kidney disease prior to study entry.

\section{Diagnoses}

Diagnoses of kidney disease (exposure), dementia (outcome), mild cognitive impairment, amnestic syndrome and covariates were based on diagnoses obtained from the Danish National Patient Registry and/ or the Danish Psychiatric Central Research Registry. These registries, covering all Danish hospitals, have recorded hospital admissions since 1977 and 1969 respectively, as well as outpatient specialist clinic visits since $1995 .^{15-17}$ We used all primary and secondary discharge diagnoses for all hospitalisations and outpatient clinic visits, but not emergency room visits (as diagnoses in this setting may be tentative and thus less valid). Diagnoses were identified according to the WHO's International Classification of Diseases 8th edition (ICD-8) until the end of 1993 and 10th edition (ICD-10) thereafter (online supplemental table 1). We used the date of hospital admission or start of outpatient clinic follow-up as the date for all diagnoses.

\section{Kidney disease}

In the main analysis, we used an extended definition of kidney disease including chronic kidney disease as well as several other persistent kidney diseases, dialysis treatment and kidney transplant (for ICD codes, see online supplemental table 1). Importantly, this extended kidney disease definition did not include acute and/or potentially reversible kidney injury. In a sensitivity analysis, we used chronic kidney disease (restricted to ICD-8 792 and ICD-10 N18) as the exposure for all-cause dementia only. Kidney Disease Improving Global Outcomes (KDIGO) defines chronic kidney as persistent ( $>3$ months) eGFR $<60 \mathrm{~mL} / \mathrm{min} / 1.73 \mathrm{~m}^{2}$ or kidney damage, often ascertained by the presence of albuminuria. ${ }^{18}$

\section{Dementia}

The validity of all-cause dementia is high with a positive predictive value of $86 \%$ in the Danish registries. ${ }^{19}$ Dementia subtypes were mutually exclusive, and we only used the first coded dementia subtype: Alzheimer's disease, vascular dementia and other (specified or unspecified) dementia, the latter comprising the majority of dementia diagnoses (for ICD codes, see online supplemental table 1). As about one-third of cases with other dementia without specification may be attributable to Alzheimer's disease, ${ }^{19}$ we also included a combined outcome of Alzheimer's disease and other dementia. 


\section{Covariates}

We identified cardiovascular disease (CVD), CVD risk factors, (any) cancer and socioeconomic status as potential confounders due to their reported associations with kidney disease and dementia (listed in table 1). ${ }^{5620} \mathrm{All}$ covariates were assessed prior to study entry. CVD covariates were angina pectoris, myocardial infarction, stroke, peripheral arterial disease, venous thromboembolism, heart failure, heart valve disease and atrial fibrillation. Covariates related to CVD risk factors were hypercholesterolaemia, hypertension, obesity, diabetes mellitus and chronic obstructive pulmonary disease as a proxy for smoking. CVD risk factors were based on diagnoses from the Danish National Patient Registry and additionally on prescriptions of lipid lowering and antihypertensive drugs (see Anatomical Therapeutic Chemical codes in online supplemental table 1) from the Danish National Prescription Registry, containing detailed individual-level data on prescriber, patient and products for all outpatient prescriptions dispensed since $1995 .{ }^{21}$

Covariates related to socioeconomic status were highest education achieved, personal gross income and employment status obtained from the Integrated Database for Labor Market Research, established in $1981 .^{22}$ Education was categorised as: low (elementary school only), medium (high school and/or academy profession degree) and high (bachelor's, master's or higher degree). Personal gross income was categorised in quartiles. Employment status was categorised as: employed, retired and unemployed. We used employment status during the 12-24 months preceding the study entry, since employment status during the year prior to kidney disease diagnosis is likely to underestimate the peak employment status.

\section{Patient and public involvement \\ No patients involved.}

\section{Statistical analysis}

We compared cumulative incidence (risk) of death as well as all-cause dementia (taking the competing risk of death into account) for the kidney disease and comparison cohorts. HRs for all-cause dementia and dementia subtypes and their corresponding 95\% CIs were calculated using Cox regression analyses with time-on-study as the time scale. Proportional hazards assumption was tested graphically by log-log plots, and no violations were detected (online supplemental figure 1). Age, sex and calendar year of index date were already controlled for in the unadjusted Cox model, as these were the matching criteria. However, to account for the matching methodology and due to the built-in selection bias (see the Discussion section) as the matching could not be completely retained, the adjusted Cox model therefore included adjustments for age (age groups listed in table 1), sex and calendar year of index date, as well as other potential confounders (as listed in table 1). Participants with missing values $(<1 \%$ of personal gross income and $<11 \%$ of employment status and education level each) were excluded from the adjusted analyses. Participants were followed from 1 year after index date until a diagnosis of dementia or censoring at 31 December 2016, emigration or death, whichever came first. Thus, the minimum follow-up time was 1 year and maximum 22 years. Because all diagnoses and vital and emigration status are registered in national registries, we had no losses to follow-up.

We performed predefined stratification analyses for age (18-49, 50-59, 60-74, 75-84 and >85 years), sex, calendar year of index date (1995-2003 or 2004-2016), CVD, CVD risk factors, socioeconomic factors and follow-up time (1-5 years, $1-10$ years and 1-22 years). Finally, in order to assess whether the risk of all-cause dementia was linked to kidney disease severity, we stratified the kidney disease cohort by presence or absence of kidney failure (defined as receiving dialysis treatment and/or kidney transplant, for codes see online supplemental table 1).

All analyses were performed using SAS V.9.4 (SAS Institute).

\section{RESULTS}

The study cohort consisted of a kidney disease cohort of 82690 patients with kidney disease and a comparison cohort of 413405 matched individuals from the general population without kidney disease. The median age was 69 years (IQR: $56-78$ years). Women comprised $41 \%$ of all participants, and $71 \%$ were enrolled during 20042016 and the remaining 29\% during 1995-2003 (table 1). Diagnoses of CVD and CVD risk factors were much more frequent in the kidney disease cohort than in the comparison cohort (table 1). Furthermore, the kidney disease cohort had lower income, higher unemployment rate and lower education than the comparison cohort (table 1). Finally, the follow-up time was shorter for the kidney disease cohort than for the comparison cohort, with a median of 3.7 and 5.2 years, respectively (table 1). This difference reflects a higher mortality rate in the kidney disease than the comparison cohort: 5-year and 10-year mortality was twice as high in patients with kidney disease compared with the general population (figure 2). During the study period, 466071 (94\%) participants died, 78555 (95\%) from the kidney disease cohort and 387516 (94\%) from the comparison cohort.

\section{Kidney disease and risk of developing dementia}

During follow-up, 3462 (4.19\% of 82690$)$ patients with kidney disease and 21879 (5.29\% of 413405$)$ individuals from the comparison cohort developed dementia, the majority classified as other dementia (table 2). Alzheimer's disease was more frequent in the comparison cohort, and vascular dementia in the kidney disease cohort (table 2).

The 5-year, 10-year and 22-year risks of all-cause dementia were lower in patients with kidney disease than in the general population: $2.90 \%$ (95\% CI $2.78 \%$ to $3.08 \%), 4.96 \%(4.79 \%$ to $5.14 \%)$ and $7.05 \%(6.70 \%$ to $7.41 \%)$ for the kidney disease cohort and $2.98 \%$ (2.92\% 
Open access

Table 1 Characteristics of study cohort at baseline

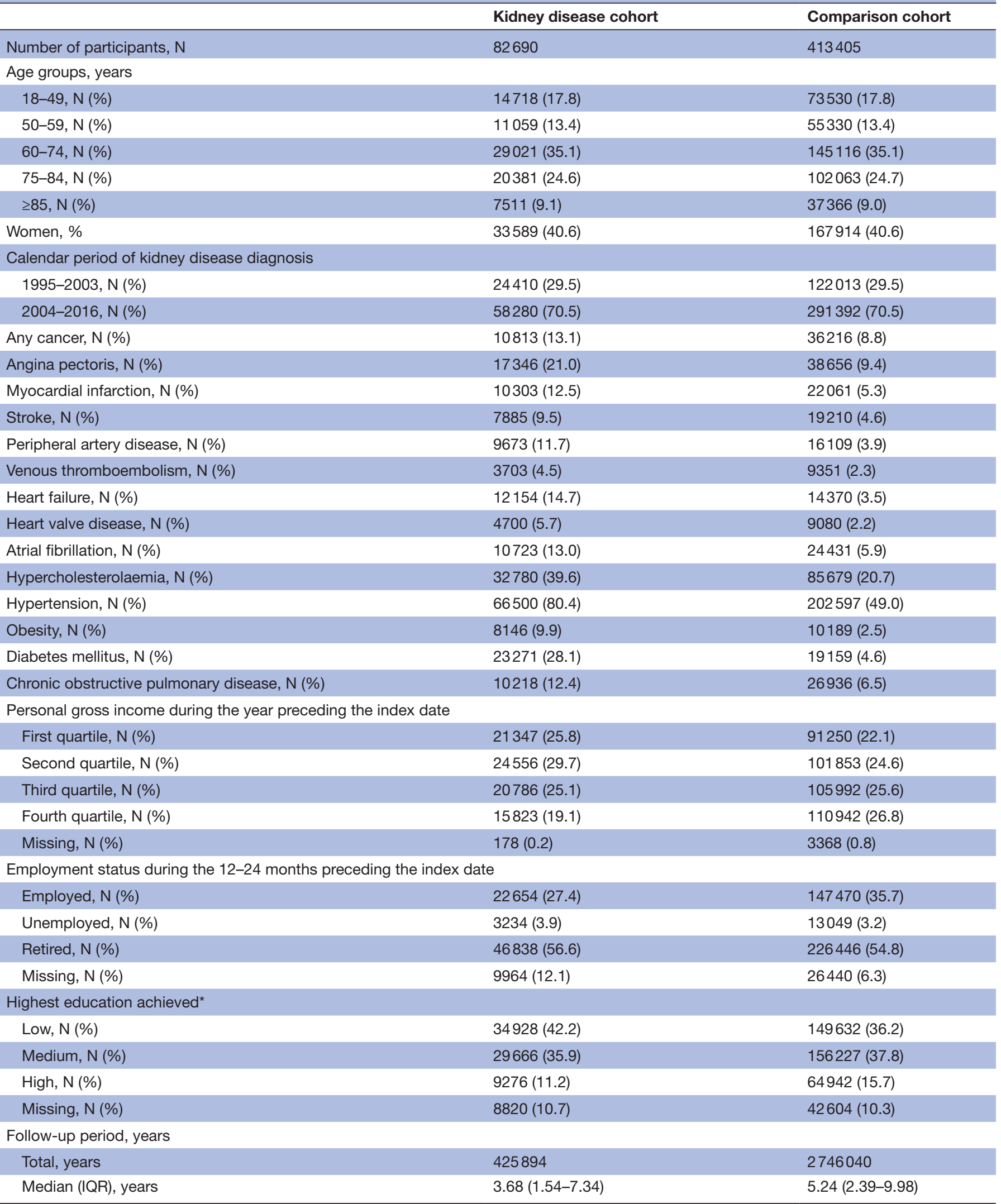

Values are expressed as numbers, frequencies, median and interquartile (IQR) values.

*Education was categorised as: low (elementary school only), medium (high school and/or academy profession degree) and high (bachelor's, master's or higher degree). 
A

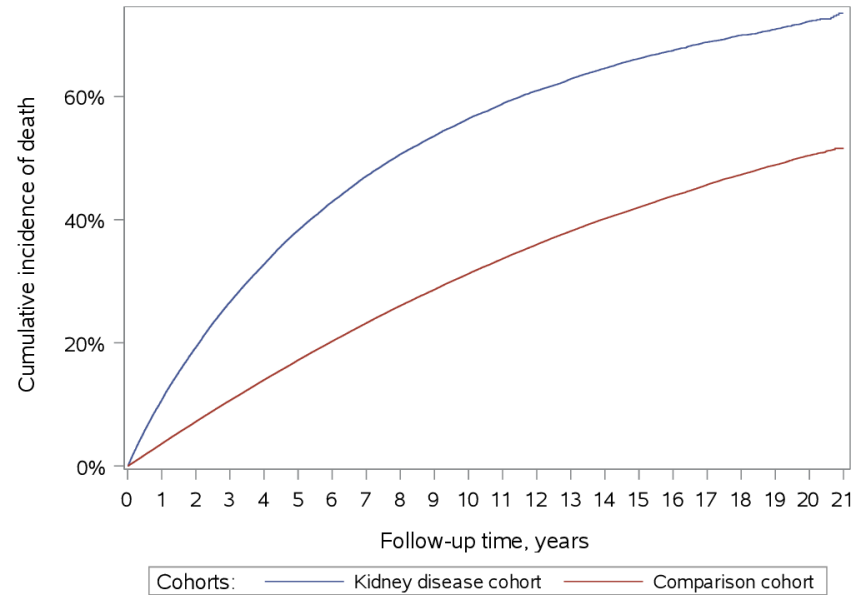

B

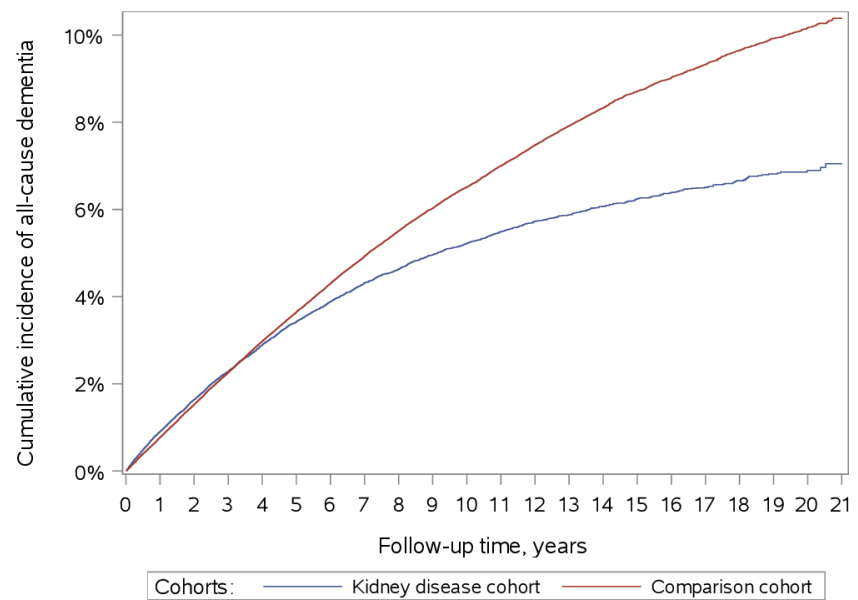

Figure 2 Cumulative incidences of $(A)$ death and (B) all-cause dementia in patients with kidney disease (kidney disease cohort) and individuals in a matched population without kidney disease (comparison cohort).

to $3.04 \%), 6.03 \%(5.94 \%$ to $6.12 \%)$ and $10.39 \%(10.17 \%$ to $10.60 \%$ ) for the comparison cohort (figure 2).

The estimates for dementia subtypes were lowest for Alzheimer's disease and highest for vascular dementia (table 2).

The adjusted HR (aHR) for all-cause dementia was stable over time. 1.06 (1.00 to 1.12) for up to 5 years of follow-up, 1.08 (1.03 to 1.13) for up to 10 years of follow-up and 1.08 (1.03 to 1.12) for up to 22 years of follow-up (table 2). When we restricted the kidney disease exposure to chronic kidney disease only, the aHR for all-cause dementia was 1.04 (0.98 to 1.10$)$ for up to 22 years of follow-up and very similar for shorter follow-up (table 2).

In analyses stratified by age, there was a stepwise decrease in HRs of all-cause dementia with increasing age: the aHRs for $18-49,50-59,60-74,75-84$ and $\geq 85$ years age groups were 1.14 (0.78 to 1.67$), 1.32$ (1.09 to 1.61$), 1.16$ (1.08 to 1.24$), 1.01$ (0.95 to 1.08 ) and 0.90 ( 0.77 to 1.04$)$, respectively. The rate of all-cause dementia did not differ by sex, calendar year of index date or socioeconomic factors. Kidney disease was also associated with increased HR for dementia in most CVD subgroups (myocardial infarction, stroke, peripheral arterial disease, venous thromboembolism, heart failure and heart valve disease) and CVD risk factors (atrial fibrillation, hypertension, obesity and diabetes mellitus), but estimates were imprecise (figure 3). Results for dementia subtypes showed consistent results (online supplemental figure 2).

\section{Kidney disease severity and risk of developing dementia}

In the kidney disease cohort, fewer patients with end-stage kidney disease developed dementia during follow-up compared with other patients with kidney disease: $3.3 \%$ (61 out of 1866) of patients with dialysis treatment or kidney transplant and 4.2\% (3401 out of 80,982) of patients without these interventions.

\section{DISCUSSION}

In this nationwide study of nearly 500000 participants, we found that being diagnosed with kidney disease is associated with a modestly increased risk of future dementia. When we restricted the exposure to chronic kidney disease only, the association was similar.

We found substantially smaller estimates than the only previous population-based study, where investigators in Taiwan found an HR of 1.41 (1.32 to 1.50) for all-cause dementia in patients with kidney disease compared with the general population. ${ }^{8}$ This may partly be explained by differences between these Asian and European populations, study design differences or both. Our study included more recent data, five times as many participants, finer age matching and a longer follow-up period. Furthermore, we included dialysis treatment, kidney transplantation and hypertensive nephropathy in our kidney disease definition, and we did not exclude participants based on other kidney-related diagnoses. In contrast, the Taiwanese study excluded patients with these and several other kidney-related diagnoses. Thus, our study likely included relatively more patients with severe kidney disease in the kidney disease cohort and mild kidney disease in the comparison cohort. Finally, while we excluded patients who were diagnosed with dementia within 1 year after kidney disease diagnosis, the Taiwanese study did not do this, and in this population, the incidence rate ratio for less than 2 years of follow-up was substantially higher than the incidence rate ratio for two or more years of follow-up. ${ }^{8}$

A meta-analysis of cross-sectional and cohort studies including more than 50000 participants showed an association between kidney disease (eGFR $<60 \mathrm{~mL} / \mathrm{min} / 1.73$ $\mathrm{m}^{2}$ ) and cognitive impairment. ${ }^{13}$ The cognitive domains that were predominantly affected (ie, orientation, attention, concept formation and reasoning) differed from those affected by dementia, suggesting that kidney disease may be more closely linked with other cognitive 


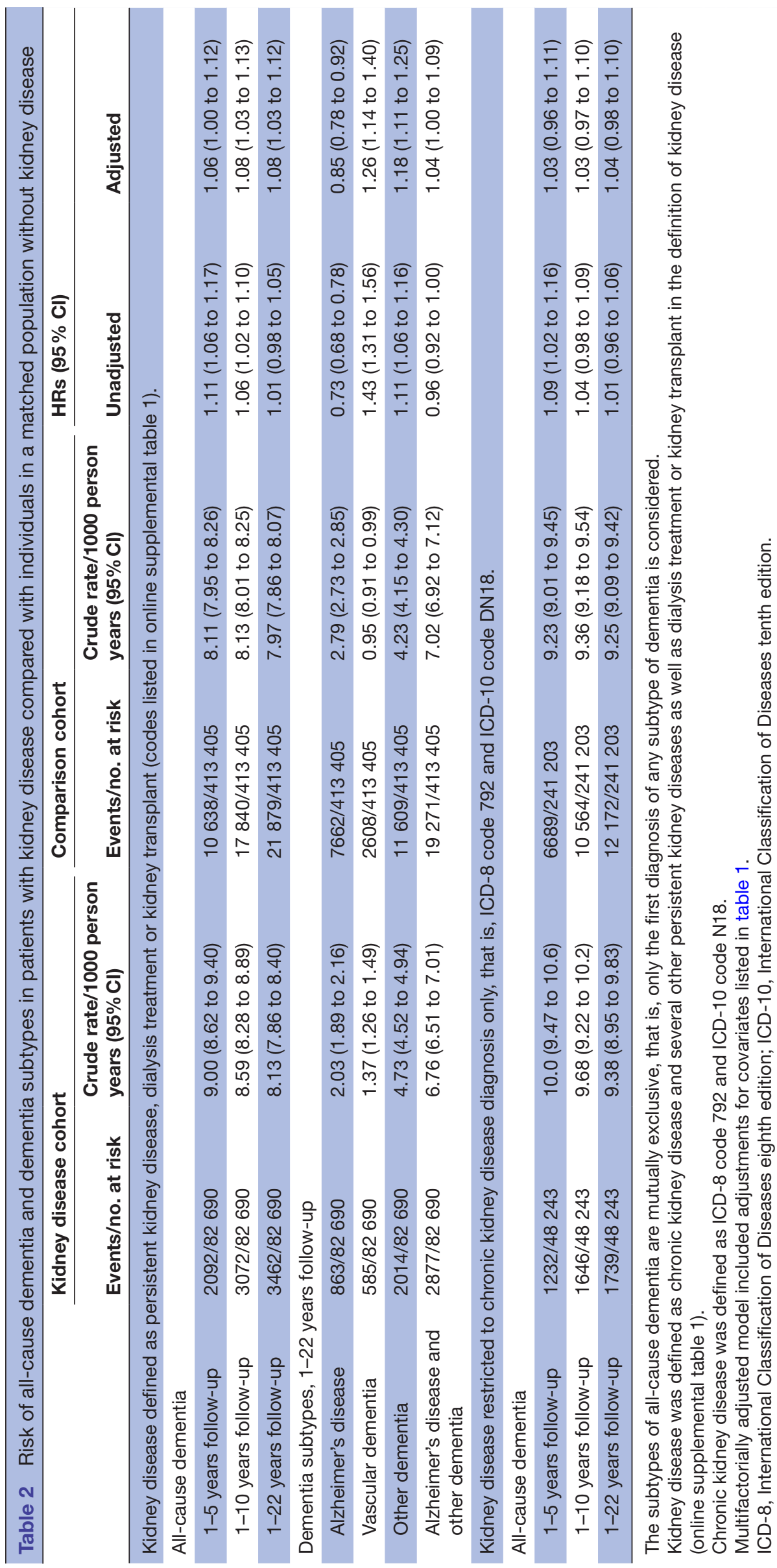




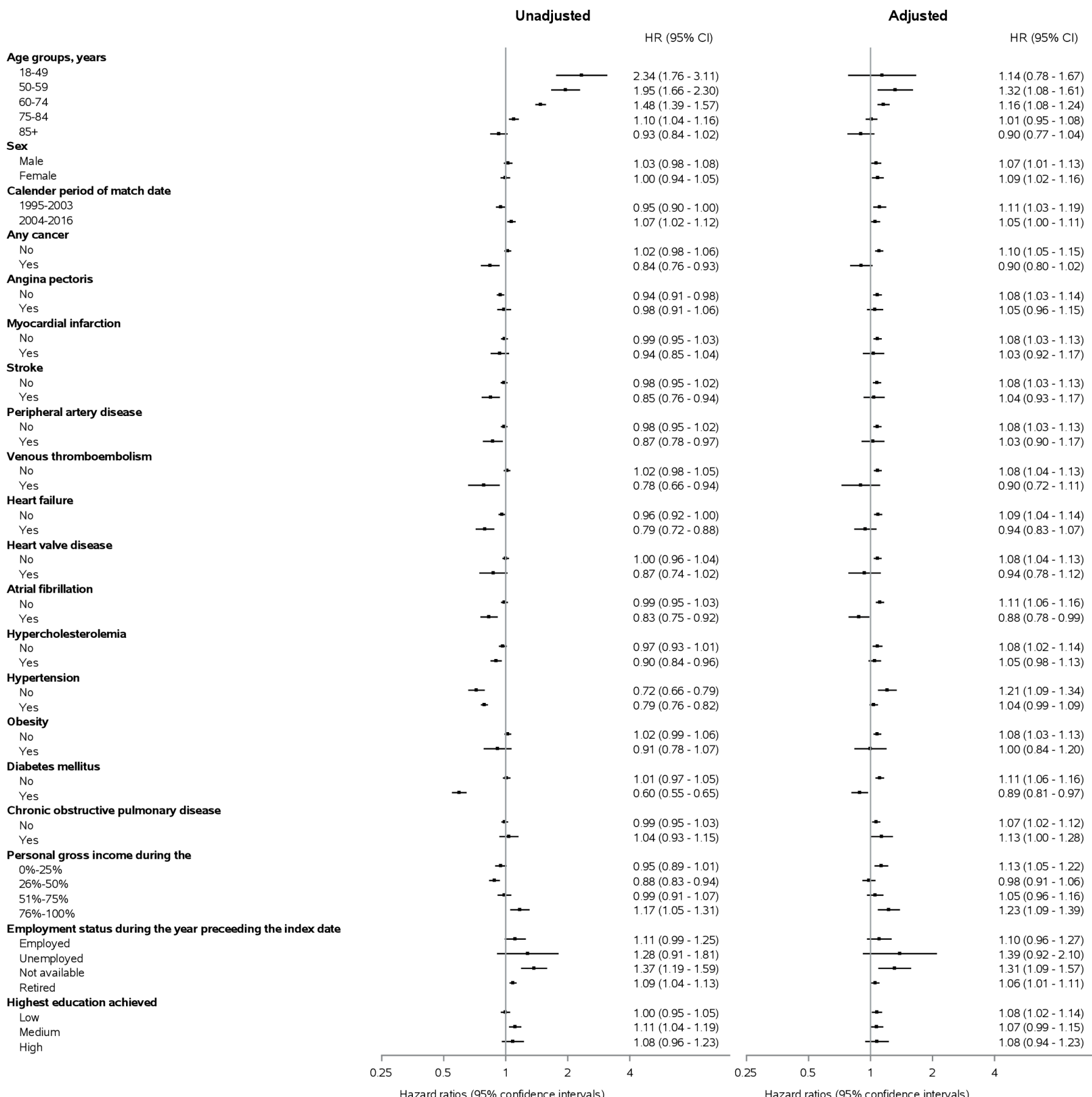

Figure 3 Risk of all-cause dementia in patients with kidney disease compared with individuals in a matched population without kidney disease stratified by covariates listed in table 1 .

impairment than with dementia. Unfortunately, we did not have data on cognitive performance. ${ }^{21} 22$

Interestingly, studies that mainly included eGFR measurements within the normal range showed a stronger association between albuminuria and dementia than between eGFR and dementia. ${ }^{9-12} 23$ This finding suggests that albuminuria may be a better marker than eGFR of more advanced kidney disease. Unfortunately, we did not have data on albuminuria or eGFR.

The lack of a strong association between kidney disease and dementia may possibly be explained in part by survivor bias due to very high mortality among patients with kidney disease. ${ }^{24}$ As dementia increases with age, patients with kidney disease may not survive long enough to develop dementia. Indeed, the fraction of participants diagnosed with dementia was lower in patients with severe than mild kidney disease $(3.3 \%$ of patients with dialysis treatment or kidney transplant vs $4.2 \%$ of patients without these interventions). This finding may reflect survivor bias or might suggest that clinicians are more likely to underdiagnose dementia in the presence of life-threatening illness and reduced life expectancy 
(detection bias). This inference is further supported by our stratification analyses, that show lower risk estimates in the presence of CVD, for example, myocardial infarction, and CVD risk factors known to be associated with increased mortality. ${ }^{25}$ In contrast, a previous Danish study of 314911 patients with myocardial infarction matched with 1573193 individuals from the general population reported that myocardial infarction was associated with higher risk of vascular dementia but not with risk of all-cause dementia or other subtypes. ${ }^{26}$ Taken together, these findings suggest a possible misclassification bias for dementia subtypes as clinicians may be more likely to diagnose vascular dementia, and less likely Alzheimer's disease, in patients with dementia and kidney disease or myocardial infarction than in individuals without these diseases.

Since HRs may change over time, the observed modest association between kidney disease and dementia may be limited to the first few years after a kidney disease diagnosis. On the other hand, the period-specific HRs are prone to a built-in selection bias. ${ }^{24}$ In our study, this translates to preferential censoring of patients, due to death, from the kidney disease cohort in the beginning of follow-up. With increasing follow-up time, this can lead to a relative increase in the proportion of individuals susceptible to dementia in the comparison cohort and thereby explain why the unadjusted HRs attenuated with increasing follow-up time. Due to this built-in selection bias, the matching could not be retained, and for this reason we included matching covariates in our adjusted analysis. This can possibly explain why the unadjusted HRs attenuated, while the aHRs did not attenuate with increasing follow-up time.

The major strength of our study is its design: large nationwide registry-based cohort study with individuallevel data and a complete follow-up on all Danish patients with hospital-diagnosed kidney disease and a matched general population comparison cohort without kidney disease during a study period from 1995 to 2016.

Limitations of our study include selection, survival and surveillance bias. As we did not perform multiple imputations for income, employment status and education level, the exclusion of participants with missing values may have biased our estimates. However, this would only bias the estimates if the missing values were not random. The unbiased estimates may be even larger if the missing values are linked to lower levels of income, employment and education. Further limitations are misclassification bias (of kidney disease, dementia and covariates), unmeasured or residual confounding, quality of coding and validity of diagnoses. The positive predictive value of kidney disease coded in the Danish National Patient Registry has been reported to be $100 \%$, whereas completeness may only be $37 \%$; that is, not all individuals with kidney disease are captured. ${ }^{27-29}$ While the positive predictive value of allcause dementia and Alzheimer's disease in the Danish National Patient Registry is $86 \%$ and $81 \%$, respectively, it is lower for other dementia subtypes. ${ }^{19}$ Thus, the results pertaining to dementia subtypes should be interpreted cautiously. This caveat is particularly important since our results are compatible with differential misclassification of dementia subtypes among patients with kidney disease, where vascular risk factors are especially common, and the general population, where vascular risk is lower. Furthermore, we used the date of hospital admission or start of outpatient clinic follow-up as the date for all diagnoses since the exact day is not available. This may have introduced a bias, particularly in the beginning of the follow-up. Additionally, there is a variable lag time between dementia onset and the date of diagnosis. Finally, since all diagnoses are recorded by hospital physicians, mild kidney disease and mild dementia treated only by a general practitioner would not be recorded unless they were also assessed in the hospital or an outpatient clinic setting.

In conclusion, patients diagnosed with kidney disease have a modestly increased risk of being diagnosed with future dementia. This association is mainly driven by diagnoses of vascular dementia, and it may be limited to the first few years after the kidney disease diagnosis. On the other hand, patients with kidney disease may be underdiagnosed with dementia due to high mortality and other comorbidities of higher priority, and the true risk of future dementia may be somewhat higher than our study suggests.

Acknowledgements We thank Thomas Bøjer Rasmussen for valuable contributions to the discussion on study design and statistical analyses.

Contributors BRJ and CFC had full access to all of the data in the study and take responsibility for the integrity of the data and accuracy of the data analysis. ADK and CFC are guarantors of the study. Concept and design: all authors. Statistical analysis: BRJ. Drafting of the manuscript: ADK. Supervision: CFC. Interpretation and critical revision of the manuscript for important intellectual content: all authors.

Funding This work was supported by a grant from the Lundbeck Foundation (grant no. R248-2017-521) and from the Independent Research Fund Denmark (grant no. 0134-00407B). ADK is funded by an unrestricted grant from Novo Nordisk (grant no. N/A). VWH is funded by a National Institutes of Health grant (grantno. P30AG066615).

Disclaimer The funding sources had no role in the design, conduct, analysis or reporting of this study.

Competing interests The Department of Clinical Epidemiology, Aarhus University Hospital, receives funding for other studies from companies in the form of research grants to (and administered by) Aarhus University.

Patient consent for publication Not applicable.

Ethics approval The study was registered at Aarhus University (record number 2016-051-000001/603) as mandated by the Danish Data Protection Agency. According to Danish legislation, registry-based studies do not require ethical review board approval or informed consent from the participants.

Provenance and peer review Not commissioned; externally peer reviewed.

Data availability statement № data are available. Data was accessed at secure servers, and cannot be shared due to Danish legislation.

Supplemental material This content has been supplied by the author(s). It has not been vetted by BMJ Publishing Group Limited (BMJ) and may not have been peer-reviewed. Any opinions or recommendations discussed are solely those of the author(s) and are not endorsed by BMJ. BMJ disclaims all liability and responsibility arising from any reliance placed on the content. Where the content includes any translated material, BMJ does not warrant the accuracy and reliability of the translations (including but not limited to local regulations, clinical guidelines, terminology, drug names and drug dosages), and is not responsible 
for any error and/or omissions arising from translation and adaptation or otherwise.

Open access This is an open access article distributed in accordance with the Creative Commons Attribution Non Commercial (CC BY-NC 4.0) license, which permits others to distribute, remix, adapt, build upon this work non-commercially, and license their derivative works on different terms, provided the original work is properly cited, appropriate credit is given, any changes made indicated, and the use is non-commercial. See: http://creativecommons.org/licenses/by-nc/4.0/.

\section{ORCID iDs}

Alisa D Kjaergaard http://orcid.org/0000-0003-3899-1324

Victor W Henderson http://orcid.org/0000-0003-1198-9240

Christian F Christiansen http://orcid.org/0000-0002-0727-953X

\section{REFERENCES}

1 Arvanitakis Z, Shah RC, Bennett DA. Diagnosis and management of dementia: review. JAMA 2019;322:1589-99.

2 Knopman DS. The enigma of decreasing dementia incidence. JAMA Netw Open 2020;3:e2011199.

3 World Alzheimer Report 2015: the global impact of dementia: an analysis of prevalence, incidence, cost and trends. volume 2020, 2015. Available: https://www.alz.co.uk/research/WorldAlzheimerRe port2015.pdf

4 GBD Chronic Kidney Disease Collaboration. Global, regional, and national burden of chronic kidney disease, 1990-2017: a systematic analysis for the global burden of disease study 2017. Lancet 2020;395:709-33.

5 Barnes DE, Yaffe K. The projected effect of risk factor reduction on Alzheimer's disease prevalence. Lancet Neurol 2011;10:819-28.

6 Ikram MA, Vernooij MW, Hofman A, et al. Kidney function is related to cerebral small vessel disease. Stroke 2008;39:55-61.

7 Bugnicourt J-M, Godefroy O, Chillon J-M, et al. Cognitive disorders and dementia in CKD: the neglected kidney-brain axis. J Am Soc Nephrol 2013;24:353-63.

8 Cheng K-C, Chen Y-L, Lai S-W, et al. Patients with chronic kidney disease are at an elevated risk of dementia: a population-based cohort study in Taiwan. BMC Nephrol 2012;13:129.

9 Deckers K, Camerino I, van Boxtel MPJ, et al. Dementia risk in renal dysfunction: a systematic review and meta-analysis of prospective studies. Neurology 2017;88:198-208.

10 Gabin JM, Romundstad S, Saltvedt I, et al. Moderately increased albuminuria, chronic kidney disease and incident dementia: the HUNT study. BMC Nephrol 2019;20:261.

11 Takae K, Hata J, Ohara T, et al. Albuminuria increases the risks for both Alzheimer disease and vascular dementia in communitydwelling Japanese elderly: the Hisayama study. J Am Heart Assoc 2018;7. doi:10.1161/JAHA.117.006693. [Epub ahead of print: 2001 2018].
12 Paterson EN, Williams MA, Passmore P, et al. Estimated glomerular filtration rate is not associated with Alzheimer's disease in a Northern Ireland cohort. J Alzheimers Dis 2017;60:1379-85.

13 Berger I, Wu S, Masson P, et al. Cognition in chronic kidney disease: a systematic review and meta-analysis. BMC Med 2016;14:206.

14 Heide-Jørgensen U, Adelborg K, Kahlert J, et al. Sampling strategies for selecting general population comparison cohorts. Clin Epidemiol 2018;10:1325-37.

15 Mors O, Perto GP, Mortensen PB. The Danish psychiatric central research register. Scand J Public Health 2011;39:54-7.

16 Schmidt M, Schmidt SAJ, Sandegaard JL, et al. The Danish national patient registry: a review of content, data quality, and research potential. Clin Epidemiol 2015;7:449-90.

17 Schmidt M, Schmidt SAJ, Adelborg K, et al. The Danish health care system and epidemiological research: from health care contacts to database records. Clin Epidemiol 2019;11:563-91.

18 Levey AS, de Jong PE, Coresh J, et al. The definition, classification, and prognosis of chronic kidney disease: a KDIGO controversies conference report. Kidney Int 2011;80:17-28.

19 Phung TKT, Andersen BB, Høgh P, et al. Validity of dementia diagnoses in the Danish Hospital registers. Dement Geriatr Cogn Disord 2007:24:220-8.

20 Webster AC, Nagler EV, Morton RL, et al. Chronic kidney disease. Lancet 2017;389:1238-52.

21 Pottegård A, Schmidt SAJ, Wallach-Kildemoes $\mathrm{H}$, et al. Data resource profile: the Danish national prescription registry. Int $J$ Epidemiol 2017;46:798-798f.

22 Petersson F, Baadsgaard M, Thygesen LC. Danish registers on personal labour market affiliation. Scand $J$ Public Health 2011;39:95-8.

23 Guerville F, De Souto Barreto P, Coley N, et al. Kidney function and cognitive decline in older adults: examining the role of neurodegeneration. J Am Geriatr Soc 2021;69:651-659.

24 Hernán MA. The hazards of hazard ratios. Epidemiology 2010;21:13-15

25 Yusuf S, Joseph P, Rangarajan S, et al. Modifiable risk factors, cardiovascular disease, and mortality in 155722 individuals from 21 high-income, middle-income, and low-income countries (PURE): a prospective cohort study. Lancet 2020;395:795-808.

26 Sundbøll J, Horváth-Puhó E, Adelborg K, et al. Higher risk of vascular dementia in myocardial infarction survivors. Circulation 2018;137:567-77.

27 Thygesen SK, Christiansen CF, Christensen S, et al. The predictive value of ICD-10 diagnostic coding used to assess Charlson comorbidity index conditions in the population-based Danish national Registry of patients. BMC Med Res Methodol 2011;11:83.

28 Winkelmayer WC, Schneeweiss S, Mogun H, et al. Identification of individuals with CKD from Medicare claims data: a validation study. Am J Kidney Dis 2005;46:225-32.

29 Vestergaard SV, Christiansen CF, Thomsen RW, et al. Identification of patients with CKD in medical databases: a comparison of different algorithms. Clin J Am Soc Nephrol 2021;16:543-51. 